\title{
THE CUBIC POLYNOMIAL DIFFERENTIAL SYSTEMS WITH TWO CIRCLES AS ALGEBRAIC LIMIT CYCLES
}

\author{
JAUME GINÉ ${ }^{1}$, JAUME LLIBRE ${ }^{2}$ AND CLAUDIA VALLS ${ }^{3}$
}

\begin{abstract}
In this paper we characterize all cubic polynomial differential systems in the plane having two circles as invariant algebraic limit cycles.
\end{abstract}

\section{Introduction And StATEMEnt of the MAIN RESUlts}

A planar polynomial differential system is a differential system of the form

$$
\begin{aligned}
& \dot{x}=P(x, y), \\
& \dot{y}=Q(x, y),
\end{aligned}
$$

where $P$ and $Q$ are real polynomials. We say that the polynomial differential system (1) has degree $n$, if $n$ is the maximum of the degrees of the polynomials $P$ and $Q$. Usually a polynomial differential system of degree 3 is denoted simply as a cubic system. The dot in (1) denotes derivative with respect to the independent variable $t$.

In this paper we want to analyze all cubic polynomial differential systems having two circles as algebraic limit cycles.

In [4] the authors proved, first that every planar polynomial vector field of degree $n$ with $n$ invariant circles is Darboux integrable without limit cycles, and second that a planar polynomial vector field of degree $n$ has at most $n-1$ invariant circles as algebraic limit cycles. So, in particular, cubic systems have at most two circles as algebraic limit cycles.

Our first result is to provide a normal form for all cubic polynomial differential systems having two circles as invariant algebraic curves.

2010 Mathematics Subject Classification. Primary 34C05.

Key words and phrases. cubic systems, limit cycles, global phase portraits, invariant ellipse, invariant algebraic curves.

${ }^{1}$ The corresponding author is Jaume Llibre. 
Consider two circles on the plane that do not intersect between them. These circles after a scaling and a rotation of the coordinates around the origin can be written, without loss of generality, as one of the following forms:

(I) $f_{1}(x, y)=x^{2}+y^{2}-1$ and $f_{2}(x, y)=x^{2}+y^{2}-r^{2}$, with $r>1$ (in this case both circles are concentric).

(II) $f_{1}(x, y)=x^{2}+y^{2}-1$ and $f_{2}(x, y)=\left(x-x_{0}\right)^{2}+y^{2}-r^{2}$ with $0<x_{0}<x_{0}+r<1$ (this is the case in which one circle, $f_{1}=0$, contains the other, $f_{2}=0$ in the bounded region that it delimits and both circles are not concentric).

(III) $f_{1}(x, y)=x^{2}+y^{2}-1$ and $f_{2}(x, y)=\left(x-x_{0}\right)^{2}+y^{2}-r^{2}$ with $x_{0}>r+1$ and $r>0$ (this is the case in which none of the circles contains the other in the bounded region that they delimit).

Given a polynomial $f=f(x, y) \in \mathbb{C}[x, y]$ we say that $f=0$ is an invariant algebraic curve of system (1) if there exists a polynomial $K=K(x, y) \in \mathbb{C}[x, y]$ called the cofactor so that

$$
P \frac{\partial f}{\partial x}+Q \frac{\partial f}{\partial y}=K f
$$

If system (1) has degree $n$ then the cofactor has degree at most $n-1$.

Theorem 1. A cubic system having the two invariant circles $f_{1}=0$ and $f_{2}=0$ as in (I) can be written as

$$
\begin{aligned}
& \dot{x}=-y\left(a_{0}+a_{1} x+a_{2} y+a_{3} x^{2}+a_{4} x y+a_{5} y^{2}\right), \\
& \dot{y}=x\left(a_{0}+a_{1} x+a_{2} y+a_{3} x^{2}+a_{4} x y+a_{5} y^{2}\right),
\end{aligned}
$$

where $a_{i} \in \mathbb{R}$ for $i=0,1, \ldots, 5$. The cofactors of $f_{1}=0$ and $f_{2}=0$ are zero.

Note that system (2) after a rescaling of time can be written as $\dot{x}=-y, \dot{y}=x$ which has the first integral $H=x^{2}+y^{2}$. Since the first integral is defined in the whole plane, this system has no limit cycles. So when the two invariant circles of a cubic system are concentric they cannot be limit cycles.

Theorem 2. A cubic system having the two invariant circles $f_{1}=0$ and $f_{2}=0$ as in (II) or in (III) can be written as

$$
\begin{aligned}
\dot{x}= & A_{1} y+A_{2} x y+A_{3} y^{2}+A_{4} x^{2} y+A_{5} x y^{2}+A_{6} y^{3}, \\
\dot{y}= & B_{0}+B_{1} x+B_{2} y+B_{3} x^{2}-A_{3} x y-B_{0} y^{2}+B_{4} x^{3} \\
& +B_{2} x^{2} y+B_{5} x y^{2}-B_{2} y^{3},
\end{aligned}
$$


where

$$
\begin{aligned}
& A_{1}=-a_{0}-\frac{a_{2}\left(1-r^{2}+x_{0}^{2}\right)}{2 x_{0}}, \quad A_{2}=a_{2}-\frac{a_{1}\left(1-r^{2}+x_{0}^{2}\right)}{2 x_{0}}, \\
& A_{3}=-\frac{a_{3}\left(1-r^{2}+x_{0}^{2}\right)}{2 x_{0}}, \quad A_{4}=a_{0}+a_{1}, \quad A_{5}=a_{3}, \quad A_{6}=a_{0}, \\
& B_{0}=-a_{0} x_{0}-\frac{a_{2}}{2}, \quad B_{1}=a_{0}-\frac{a_{1}}{2}+\frac{a_{2}\left(1-r^{2}+x_{0}^{2}\right)}{2 x_{0}}, \quad B_{2}=-\frac{a_{3}}{2}, \\
& B_{3}=a_{0} x_{0}-\frac{a_{2}}{2}+\frac{a_{1}\left(1-r^{2}+x_{0}^{2}\right)}{2 x_{0}}, \quad B_{4}=-a_{0}-\frac{a_{1}}{2}, \quad B_{5}=-a_{0}+\frac{a_{1}}{2},
\end{aligned}
$$

with $a_{0}, a_{1}, a_{2}, a_{3} \in \mathbb{R}$. The cofactors of $f_{1}=0$ and $f_{2}=0$ are

$$
K_{1}=y\left(a_{2}+2 a_{0} x_{0}+a_{1} x+a_{3} y\right), \quad K_{2}=y\left(a_{2}+a_{1} x+a_{3} y\right),
$$

respectively.

Note that when $a_{0}=0$ system (3) has the rational first integral $f_{1} / f_{2}$, and consequently no limit cycles. Therefore in what follows we consider that $a_{0} \neq 0$.

Now we want to characterize when system (3) has $f_{1}=0$ and $f_{2}=0$ as limit cycles.

The following result shows that the two circles $f_{1}=f_{2}=0$ are never both limit cycles of system (3) when $a_{3}=0$.

Theorem 3. If $a_{3}=0$ and $a_{0} \neq 0$, then both circles $f_{1}=0$ and $f_{2}=0$ are not limit cycles of system (3).

By Theorem 3 in order that both $f_{1}=0$ and $f_{2}=0$ are limit cycles of system (3) we must have $a_{3} \neq 0$. The following result characterizes when the circles $f_{1}=0$ and $f_{2}=0$ are limit cycles of system (3). This is the main result of this paper because it characterizes when a cubic polynomial differential system has two circles as invariant algebraic limit cycles.

Theorem 4. The two circles $f_{1}=0$ and $f_{2}=0$ are limit cycles for system (3) if and only if

$$
\begin{aligned}
& a_{3} a_{0} \neq 0, \\
& \Delta_{0}=a_{3}^{2}+a_{1}^{2}-a_{2}^{2}<0, \\
& \Delta_{1}=\left(a_{1}^{2}+a_{3}^{2}\right) r^{2}-\left(a_{2}+\left(2 a_{0}+a_{1}\right) x_{0}\right)^{2}<0, \\
& \text { either } B_{4}^{2}+B_{3}^{2}+B_{1}^{2} \neq 0 \text { if (II) holds, or if (III) holds with } \\
& B_{4}=0 \text { then } B_{3} \neq 0 \text { and } B_{1}^{2}-4 B_{0} B_{3}>0 .
\end{aligned}
$$

The paper has been divided as follows: In section 2 we prove Theorems 1 and 2 . In section 3 we give some preliminary results related to system (3) that will be used in the proofs of Theorems 3 and 4 . The 
study of the singular points of system (3) is given in section 4. Finally, Theorems 3 and 4 are proved in section 5 .

Taking into account the results on algebraic limit cycles and algebraic circle limit cycles of the papers

\section{Proofs of Theorems 1 And 2}

Proof of Theorems 1 and 2. Suppose that a cubic system in the plane has two invariant circles that do not intersect. As pointed out in the introduction, two circles on the plane that do not intersect after a rescaling and a rotation of the coordinates around the origin they can be written, without loss of generality, as in (I), or as in (II), or as in (III).

We write a cubic planar polynomial differential system in the form

$$
\dot{x}=\sum_{i+j=0}^{3} a_{i j} x^{i} y^{j}, \quad \dot{y}=\sum_{i+j=0}^{3} b_{i j} x^{i} y^{j},
$$

where $a_{i j}, b_{i j} \in \mathbb{R}$ for $i+j=0,1,2,3$.

We assume that $f_{1}=0$ and $f_{2}=0$ are invariant algebraic curves of system (4), with cofactors $k_{1}$ and $k_{2}$ given, respectively, by

$$
k_{1}=\sum_{i+j=0}^{2} \alpha_{i j} x^{i} y^{j}, \quad k_{2}=\sum_{i+j=0}^{2} \beta_{i j} x^{i} y^{j},
$$

where $\alpha_{i j}, \beta_{i j} \in \mathbb{R}$ for $i+j=0,1,2$.

We recall that $f_{1}=0$ and $f_{2}=0$ are invariant algebraic curves of system (4) that is, they satisfy

$$
\begin{aligned}
k_{i} f_{i} & =\frac{\partial f_{i}}{\partial x} \dot{x}+\frac{\partial f_{i}}{\partial y} \dot{y} \\
& =\sum_{i+j=0}^{3} a_{i j} x^{i} y^{j} \frac{\partial f_{i}}{\partial x}+\sum_{i+j=0}^{3} b_{i j} x^{i} y^{j} \frac{\partial f_{i}}{\partial y}, \quad i=1,2 .
\end{aligned}
$$

If $f_{1}$ and $f_{2}$ are as in (I) from (5) we get system (2) where we have used the notation $a_{0}=a_{01}=b_{10}, a_{1}=a_{11}=b_{20}, a_{2}=a_{02}=b_{11}$, $a_{3}=a_{31}=b_{30}, a_{4}=a_{12}=b_{21}$ and $a_{5}=a_{30}=b_{12}$. Moreover, in this case $K_{1}=K_{2}=0$.

If $f_{1}$ and $f_{2}$ are as in case (II) or (III) from (5) we get system (3) with the corresponding cofactors, where we have used the notation $a_{0}=a_{03}$, $a_{1}=\beta_{11}, a_{2}=\beta_{01}$ and $a_{3}=\beta_{02}$. 


\section{Preliminary Results on System (3)}

In this section we introduce some preliminary results on system (3) that will be used in the proof of Theorems 3 and 4 .

Lemma 5. System (3) with $a_{3}=0$ is invariant with respect to the change $(x, y, t) \rightarrow(x,-y,-t)$.

Proof. System (3) with $a_{3}=0$ becomes

$$
\begin{aligned}
& \dot{x}=A_{1} y+A_{2} x y+A_{4} x^{2} y+A_{6} y^{3}, \\
& \dot{y}=B_{0}+B_{1} x+B_{3} x^{2}-B_{0} y^{2}+B_{4} x^{3}+B_{5} x y^{2}
\end{aligned}
$$

which is obviously invariant with respect to the change $(x, y, t) \rightarrow$ $(x,-y,-t)$.

Lemma 6. If system (3) has a unique singular point $p$ inside the circle $f_{1}=0$ (resp. the circle $f_{2}=0$ ) which is a limit cycle, then this singular point cannot be a center.

Proof. We will prove that if the unique singular point inside $f_{1}=0$ is a center, then $f_{1}=0$ is not a limit cycle. The proof for the circle $f_{2}$ can be done in a similar way.

Consider a Poincaré map defined in a transversal section with endpoints the center and a point of the circle $f_{1}=0$. This Poincaré map is analytic because the differential system is polynomial and consequently analytic. We have a continuum of periodic orbits surrounding the center, so the Poincaré map in a neighborhood of the center is the identity, and by analyticity it is the identity in all the consider transversal section, consequently the circle $f_{1}=0$ is not a limit cycle.

We shall need the following result. For the proof of Lemma 7 see for instance [5].

Lemma 7. Consider a two-dimensional autonomous polynomial differential system having an invariant algebraic curve $g=0$ with cofactor $k$. Then all singular points of the differential system are contained in the union of the sets $\{g=0\} \cup\{k=0\}$.

Proposition 8. The two circles $f_{1}=0$ and $f_{2}=0$ are periodic solutions for system (3) with $a_{0} \neq 0$ if and only if

(6) $\Delta_{0}=a_{3}^{2}+a_{1}^{2}-a_{2}^{2}<0, \quad \Delta_{1}=\left(a_{1}^{2}+a_{3}^{2}\right) r^{2}-\left(a_{2}+\left(2 a_{0}+a_{1}\right) x_{0}\right)^{2}<0$.

Proof. Note that $f_{1}=0$ and $f_{2}=0$ are invariant algebraic curves of system (3) with cofactors $K_{1}$ and $K_{2}$, respectively. In view of Lemma 7 , the singular points of system (3) are on $\left\{f_{1}=0\right\} \cup\left\{K_{1}=0\right\}$ and on $\left\{f_{2}=0\right\} \cup\left\{K_{2}=0\right\}$. 
We first compute the singular points on $f_{1}=0$. We obtain the four points

$$
z_{1,2}=\left(x_{1,2}, y_{1,2}\right), \quad z_{3,4}=\left(x_{3,4}, y_{3,4}\right)
$$

where

$$
x_{1,2}=\frac{-a_{2} a_{1} \pm a_{3} \sqrt{\Delta_{0}}}{a_{1}^{2}+a_{3}^{2}}, \quad y_{1,2}=\frac{-a_{2} a_{3} \mp a_{1} \sqrt{\Delta_{0}}}{a_{1}^{2}+a_{3}^{2}}
$$

and

$$
x_{3}=x_{4}=\frac{1-r^{2}+x_{0}^{2}}{2 x_{0}}, \quad y_{3,4}=\mp \sqrt{1-\frac{\left(1-r^{2}+x_{0}^{2}\right)^{2}}{4 x_{0}^{2}}} .
$$

Computing the singular points on $f_{2}=0$ we obtain the points $z_{3}=$ $\left(x_{3}, y_{3}\right), z_{4}=\left(x_{4}, y_{4}\right)$ given in (8) and two additional points

$$
z_{5,6}=\left(x_{5,6}, y_{5,6}\right)
$$

where

$$
\begin{aligned}
x_{5,6} & =\frac{-a_{2} a_{1}-2 a_{0} a_{1} x_{0}+a_{3}^{2} x_{0} \mp a_{3} \sqrt{\Delta_{1}}}{a_{1}^{2}+a_{3}^{2}}, \\
y_{5,6} & =\frac{-a_{2} a_{3}-2 a_{0} a_{3} x_{0}-a_{1} a_{3} x_{0} \pm a_{3} \sqrt{\Delta_{1}}}{a_{1}^{2}+a_{3}^{2}} .
\end{aligned}
$$

Note that the two singular points $z_{3}$ and $z_{4}$ are on the circles $f_{1}=f_{2}=$ 0 and thus they are complex.

By the expressions of $K_{1}$ and $K_{2}$ given in Theorem 2 we have that $K_{1}=y \tilde{k}$ being $\tilde{k}=a_{2}+a_{1} x+2 a_{0} x_{0}+a_{3} y$ and $K_{2}=y \hat{k}$ being $\hat{k}=$ $a_{2}+a_{1} x+a_{3} y$. Computing the singular points of system (3) on $\tilde{k}=0$ we get the points $z_{5}=\left(x_{5}, y_{5}\right), z_{6}=\left(x_{6}, y_{6}\right)$ given in (9). So, these singular points are on $f_{2}=0$. Now computing the singular points of (3) on $\hat{k}=0$ we get the points $z_{1}=\left(x_{1}, y_{1}\right)$ and $z_{2}=\left(x_{2}, y_{2}\right)$ given in (7). So, these singular points are on $f_{1}=0$. Finally, on $y=0$ we have that $K_{1}=K_{2}=0$. Moreover, computing the singular points $(\bar{x}, \bar{y})$ of (3) on $y=0$ we obtain that they satisfy

$$
F(x)=B_{0}+B_{1} \bar{x}+B_{3} \bar{x}^{2}+B_{4} \bar{x}^{3}=0 .
$$

In summary, conditions (6) together with the condition

$$
\begin{aligned}
& F(1) F(-1) F\left(x_{0}-r\right) F\left(x_{0}+r\right)= \\
& \left(a_{1}^{2}-a_{2}^{2}\right)\left(a_{2}+2 a_{0} x_{0}+a_{1}\left(r+x_{0}\right)\right) \neq 0
\end{aligned}
$$

are equivalent to say that there are no singular points on the two circles $f_{1}=0$ and $f_{2}=0$ (we recall that $f_{1}=f_{2}=0$ on $y=0$ are, respectively, 
$x= \pm 1$ and $x=x_{0} \pm r$ ). Note that (10) is automatically satisfied since condition $\Delta_{0}<0$ implies that

$$
a_{1}^{2}-a_{2}^{2}<a_{3}^{2}+a_{1}^{2}-a_{2}^{2}=\Delta_{0}<0
$$

and condition $\Delta_{1}<0$ implies that

$$
\begin{aligned}
& \left(a_{2}+2 a_{0} x_{0}+a_{1}\left(r+x_{0}\right)\right)\left(a_{2}+2 a_{0} x_{0}+a_{1} x_{0}-a_{1} r\right) \\
& =\left(a_{2}+2 a_{0} x_{0}+a_{1} x_{0}\right)^{2}-a_{1}^{2} r^{2} \\
& >\left(a_{2}+2 a_{0} x_{0}+a_{1} x_{0}\right)^{2}-\left(a_{1}^{2}+a_{3}^{2}\right) r^{2}=-\Delta_{1}>0
\end{aligned}
$$

and consequently $a_{2}+2 a_{0} x_{0}+a_{1}\left(r+x_{0}\right) \neq 0$.

\section{The SINGUlar POINTS}

In this section we study the singular points of system (3). For this, we introduce some notation.

$$
\begin{aligned}
\left.\dot{y}\right|_{y=0} & =F(x)=B_{0}+B_{1} x+B_{3} x^{2}+B_{4} x^{3}, \\
\Delta_{2} & =\left(r-x_{0}+1\right)\left(r-x_{0}-1\right)\left(r+x_{0}-1\right)\left(r+x_{0}+1\right), \\
D_{0} & =-B_{1}^{2} B_{3}^{2}+4 B_{0} B_{3}^{3}+4 B_{1}^{3} B_{4}-18 B_{0} B_{1} B_{3} B_{4}+27 B_{0}^{2} B_{4}^{2}, \\
D_{1} & =B_{3}^{2}-3 B_{4} B_{1} .
\end{aligned}
$$

First we note that by the definition of the forms of $f_{1}$ and $f_{2}$ in cases (II) and (III) we readily have that $\Delta_{2} \neq 0$. Moreover, under the assumptions $\Delta_{0}<0, \Delta_{1}<0$ we have already proved that the unique singular points of system (3) are on $y=0$ and $F(x)=0$. Now we will study them under the assumptions $\Delta_{0}<0, \Delta_{1}<0$.

We first recall some important observation that will be used all through the paper.

Lemma 9. Consider system (3) with $\Delta_{0}<0, \Delta_{1}<0$ and $a_{0} \neq 0$. Its Jacobian matrix on each of its singular points is of the form

$$
\begin{aligned}
L(x) & =\left.\left(\begin{array}{ll}
\frac{\partial \dot{x}}{\partial x} & \frac{\partial \dot{x}}{\partial y} \\
\frac{\partial \dot{y}}{\partial x} & \frac{\partial \dot{y}}{\partial y}
\end{array}\right)\right|_{y=0} \\
& =\left(\begin{array}{cc}
0 & A_{1}+A_{2} x+A_{4} x^{2} \\
B_{1}+2 B_{3} x+3 B_{4} x^{2} & B_{2}-A_{3} x+B_{2} x^{2}
\end{array}\right),
\end{aligned}
$$

with $A_{1}+A_{2} x+A_{4} x^{2} \neq 0$. Moreover, if $a_{3} \neq 0$ then $B_{2}-A_{3} x+B_{2} x^{2} \neq 0$. 
Proof. Compute $L(x)$ as in (12). Doing the resultant between $A_{1}+$ $A_{2} x+A_{4} x^{2}$ and $F(x)$ with respect to $x$ we get

$$
\begin{aligned}
& -\frac{a_{0}}{16 x_{0}^{2}}\left(a_{1}^{2}-a_{2}^{2}\right)\left(a_{2}+2 a_{0} x_{0}+a_{1} x_{0}+a_{1} r\right)\left(a_{2}+2 a_{0} x_{0}+a_{1} x_{0}-a_{1} r\right) \Delta_{2} \\
& =\frac{a_{0}}{16 x_{0}^{2}} \tilde{\Delta}_{0} \tilde{\Delta}_{1} \Delta_{2},
\end{aligned}
$$

where $\tilde{\Delta}_{0}$ and $\tilde{\Delta}_{1}$ are, respectively, $\Delta_{0}$ and $\Delta_{1}$ restricted to $a_{3}=0$. By assumptions we have $\Delta_{0}<0, \Delta_{1}<0, a_{0} \neq 0$ and by definition $\Delta_{2} \neq 0$. So, $A_{1}+A_{2} x+A_{4} x^{2} \neq 0$ at the singular points.

If $a_{3} \neq 0$ doing the resultant between $B_{2}-A_{3} x+B_{2} x^{2}$ and $F(x)$ with respect to $x$ we get

$$
\frac{a_{0}^{2} a_{3}^{2} r^{2} \Delta_{2}}{8 x_{0}^{2}}
$$

which is different from zero because by assumptions $a_{0} \neq 0$ and by definition $\Delta_{2} x_{0} \neq 0$. So, when $a_{3} \neq 0, B_{2}-A_{3} x+B_{2} x^{2} \neq 0$ at the singular points.

When $a_{3}=0$ then $B_{2}=A_{3}=0$ and in view of Lemma 9 the singular points can be either hyperbolic, or nilpotent but not linearly zero. In the next result we study the singular points of system (3) when $a_{3}=0$. In this case system (3) becomes

$$
\begin{aligned}
& \dot{x}=A_{1} y+A_{2} x y+A_{4} x^{2} y+A_{6} y^{3}, \\
& \dot{y}=F(x)-B_{0} y^{2}+B_{5} x y^{2} .
\end{aligned}
$$

Lemma 10. Consider system (3) with $a_{3}=0, \Delta_{0}<0, \Delta_{1}<0$ and $a_{0} \neq 0$ If $\tilde{x}$ is a simple solution of $F(x)=0$, then the singular point $(\tilde{x}, 0)$ is either a saddle or a center. If $\tilde{x}$ is a multiple solution of $F(x)=0$ (either double or triple), then the singular point $(\tilde{x}, 0)$ will be nilpotent.

Proof. In view of Lemma 9, the Jacobian matrix at a singular point $(\tilde{x}, 0)$ with $F(\tilde{x})=0$ is of the form (12) with $A_{1}+A_{2} \tilde{x}+A_{4} \tilde{x}^{2} \neq 0$.

If $\tilde{x}$ is a simple solution of $F(x)=0$ then $B_{1}+2 B_{3} \tilde{x}+3 B_{4} \tilde{x}^{2}=$ $d F(x) /\left.d x\right|_{x=\tilde{x}} \neq 0$. Therefore, the singular point $(\tilde{x}, 0)$ will be either a saddle, or a focus, or a center. Translating the singular point at the origin, by making the change $X=x-\tilde{x}, Y=y$ we see that system (13) in the variables $(X, Y)$ is reversible (see Lemma 5 ), and so the singular point $(\tilde{x}, 0)$ will be either a saddle, or a center. This proves the first statement in the lemma. 
If $\tilde{x}$ is a multiple solution of $F(x)=0$, then $B_{1}+2 B_{3} \tilde{x}+3 B_{4} \tilde{x}^{2}=$ $d F(x) /\left.d x\right|_{x=\tilde{x}}=0$, then the singular point $(\tilde{x}, 0)$ will be nilpotent. This proves the lemma.

When $B_{4} \neq 0$ we will write $F(x)=B_{4} G(x)$ and we will talk about the zeros of $G(x)=0$.

We shall need the following result which is the part of the Andreev's theorem for nilpotent singular points [1], see also Theorem 3.5 in [2].

Lemma 11. Let $(0,0)$ be an isolated nilpotent singular point of the vector field $X$ given by

$$
\dot{x}=y+A(x, y), \quad \dot{y}=B(x, y)
$$

where $A, B$ are analytic in a neighborhood of $(0,0)$ and also $A_{x}(0,0)=$ $A_{y}(0,0)=B_{x}(0,0)=B_{y}(0,0)=0$. Let $y=f(x)$ be the solution of $y+$ $A(x, y)=0$ in a neighborhood of $(0,0)$ and consider $F(x)=B(x, f(x))$ and $G(x)=(\partial A / \partial x+\partial B / \partial y)(x, f(x))$. Assume $F(x)=a x^{m}+o\left(x^{m}\right)$ with $a \neq 0$ and $G(x)=0$. If $m$ is odd and $a>0$, then $(0,0)$ is a saddle. If $m$ is odd and $a<0$ then $(0,0)$ is a focus, or a center. If $m$ is even then $(0,0)$ is a cusp.

Lemma 12. System (3) with $a_{3}=0, a_{0} \neq 0$ and $\Delta_{1}<0, \Delta_{2}<0$ has the following singular points:

(a) If $B_{4}=0$ and $B_{3}=0$ then if $B_{1}=0$ it has no singular points and if $B_{1} \neq 0$ it has the unique singular point $\left(z_{0}, 0\right)$ where

$$
z_{0}=-\frac{B_{0}}{B_{1}}
$$

and it is either a saddle or a center.

(b) Assume $B_{4}=0$ and $B_{3} \neq 0$. If (II) holds, or (III) holds with $B_{1}^{2}-4 B_{0} B_{3}>0$, then it has the two singular points $\left(z_{1}, 0\right)$ and $\left(z_{2}, 0\right)$ with

$$
z_{1,2}=\frac{-B_{1} \pm \sqrt{B_{1}^{2}-4 B_{0} B_{3}}}{2 B_{3}} .
$$

Both singular points are either saddles or centers. If (III) holds with $B_{1}^{2}-4 B_{0} B_{3} \leq 0$, then either $f_{1}=0$, or $f_{2}=0$ cannot be a limit cycle.

(c) If $B_{4} \neq 0, D_{0}=D_{1}=0$, then it has a unique multiple real solution $z_{3}$ of $G(x)=0$. The singular point $\left(z_{3}, 0\right)$ is either a saddle or a center.

(d) If $B_{4} \neq 0, D_{0}=0$ and $D_{1} \neq 0$, then $G(x)=0$ has two distinct real solutions (one simple $z_{4}$ and one double $z_{5}$ ). The singular 
point $\left(z_{4}, 0\right)$ is a saddle or center and the singular point $\left(z_{5}, 0\right)$ is a cusp.

(e) If $B_{4} \neq 0$ and $D_{0}<0$, then $G(x)=0$ has three distinct simple real solutions $z_{6}, z_{7}, z_{8}$. All of them can only be saddles or centers.

(f) If $B_{4} \neq 0$ and $D_{0}>0$, then $G(x)=0$ has a unique simple real solution $z_{9}$. The singular point $\left(z_{9}, 0\right)$ is either a saddle or a center.

Proof. It follows by direct calculations. Note that when $B_{4}=0$ equation $F(x)=0$ becomes the quadratic equation $B_{3} x^{2}+B_{1} x+B_{0}=0$.

If $B_{3}=0$ then if $B_{1}=0$ it has no solutions and if $B_{1} \neq 0$, then the solution is as given in (15). It is a simple solution of $F(x)=0$ and by Lemma 10 it is either a saddle or a center. This proves statement (a).

If $B_{4}=0$ and $B_{3} \neq 0$ then $F(x)=0$ has the two solutions (16), where

$$
B_{1}^{2}-4 B_{0} B_{3}=\frac{16 a_{0}^{2} r^{2} x_{0}^{2}+a_{2}^{2} \Delta_{2}}{4 x_{0}^{2}} .
$$

It is easy to see that if we are under the assumptions (II) then $\Delta_{2}>0$ and if we are under the assumptions (III) then $\Delta_{2}<0$. So, under the assumptions (II) the solutions $z_{1}$ and $z_{2}$ are simple because $B_{1}^{2} \neq$ $4 B_{0} B_{3}>0$ and by Lemma 10 both solutions can only be saddles or centers. Under the conditions (III), if $B_{1}^{2}-4 B_{0} B_{3}>0$ then again $z_{1}$ and $z_{2}$ are simple and they are either saddles or centers. On the other hand if $B_{1}^{2}-4 B_{0} B_{3} \leq 0$ then either we have no singular points or one singular point. But in this case since none of the circles $f_{1}=0$ and $f_{2}=0$ contains the other in the bounded region that they delimit, and inside one limit cycle there must be a singular point, we conclude that either $f_{1}=0$, or $f_{2}=0$ cannot be a limit cycle. This proves statement (b).

If $B_{4} \neq 0$ and $D_{0}=D_{1}=0$ we have a unique triple solution for $G(x)=0$, that we call $z_{3}$. This means that $F(x)=B_{4}\left(x-z_{3}\right)^{3}$. With a parametrization of the time we can rewrite equation (13) as

$$
\begin{aligned}
& \dot{x}=\tilde{A}_{1} y+\tilde{A}_{2} x y+\tilde{A}_{4} x^{2} y+\tilde{A}_{6} y^{3}, \\
& \dot{y}=\left(x-z_{3}\right)^{3}-\tilde{B}_{0} y^{2}+\tilde{B}_{5} x y^{2},
\end{aligned}
$$

where $\tilde{A}_{i}=A_{i} / B_{4}$ and $\tilde{B}_{j}=B_{j} / B_{4}$ for $i=1,2,4,6$ and $j=0,5$. We introduce the change of variables $X=x-z_{3}$ and $Y=y$. Then system 
(17) becomes

$$
\begin{aligned}
& \dot{x}=\hat{A}_{1} Y+\hat{A}_{2} X Y+\hat{A}_{4} X^{2} Y+\tilde{A}_{6} Y^{3}, \\
& \dot{y}=X^{3}-\hat{B}_{0} y^{2}+\tilde{B}_{5} X Y^{2},
\end{aligned}
$$

where $\hat{A}_{1}=\tilde{A}_{1}+\tilde{A}_{2} z_{3}+\tilde{A}_{4} z_{3}^{2}, \hat{A}_{2}=\tilde{A}_{2}+2 \tilde{A}_{4} z_{3}$ and $\hat{B}_{0}=B_{0}-B_{5} z_{3}$. Note that in view of Lemma 9 we have that $\hat{A}_{1} \neq 0$. Now we introduce a scaling of time of the form $\tau=t / \hat{A}_{1}$ and we have that system (18) becomes

$$
\begin{aligned}
& \dot{x}=Y+\bar{A}_{2} X Y+\bar{A}_{4} X^{2} Y+\bar{A}_{6} Y^{3}, \\
& \dot{y}=\frac{1}{\hat{A}_{1}} X^{3}-\bar{B}_{0} Y^{2}+\bar{B}_{5} X Y^{2},
\end{aligned}
$$

where

$$
\bar{A}_{2}=\frac{\hat{A}_{2}}{\hat{A}_{1}}, \bar{A}_{4}=\frac{\hat{A}_{4}}{\hat{A}_{1}}, \bar{A}_{6}=\frac{\tilde{A}_{6}}{\hat{A}_{1}}, \bar{B}_{0}=\frac{\hat{B}_{0}}{\hat{A}_{1}}, \bar{B}_{5}=\frac{\tilde{B}_{5}}{\hat{A}_{1}} .
$$

Note that $(0,0)$ is a nilpotent singular point and that system (19) is precisely system (14) with

$$
\begin{aligned}
& A(X, Y)=\bar{A}_{2} X Y+\bar{A}_{4} X^{2} Y+\bar{A}_{6} Y^{3}, \\
& B(X, Y)=\frac{1}{\hat{A}_{1}} X^{3}-\bar{B}_{0} Y^{2}+\bar{B}_{5} X Y^{2} .
\end{aligned}
$$

The solution $Y+f(X)$ of $Y+A(X, Y)=0$ is $f(X)=0$. Then $F(X)=$ $B(X, 0)=\frac{1}{\hat{A}_{1}} X^{3}$ and $G(X)=\left(A_{2}+2 A_{4} X-2 B_{0} Y+2 B_{5} X Y\right)_{Y=0}=0$. So we are under the assumptions of Lemma 11 with $m=3$ and $a=$ $1 / \hat{A}_{1}$. In view of Lemma 11 we have that it is either a saddle or a focus or a center. Since the system is reversible it can only be a saddle or a center. This proves statement (c).

If $B_{4} \neq 0$ and $D_{0}=0$ and $D_{1} \neq 0$ we have a single solution and a double solution for $G(x)=0$, that we call respectively $z_{4}$ and $z_{5}$. The solution $z_{4}$ is simple and thus by Lemma 10 it is either a saddle or a center.

The solution $z_{5}$ is double. This means that $F(x)=B_{4}\left(x-z_{4}\right)(x-$ $\left.z_{5}\right)^{2}$. With a parametrization of the time we can rewrite equation (13) as

$$
\begin{aligned}
& \dot{x}=\tilde{A}_{1} y+\tilde{A}_{2} x y+\tilde{A}_{4} x^{2} y+\tilde{A}_{6} y^{3}, \\
& \dot{y}=\left(x-z_{4}\right)\left(x-z_{5}\right)^{2}-\tilde{B}_{0} y^{2}+\tilde{B}_{5} x y^{2},
\end{aligned}
$$

where $\tilde{A}_{i}=A_{i} / B_{4}$ and $\tilde{B}_{j}=B_{j} / B_{4}$ for $i=1,2,4,6$ and $j=0,5$. We introduce the change of variables $X=x-z_{5}$ and $Y=y$. Then system 
(20) becomes

$$
\begin{aligned}
& \dot{x}=\hat{A}_{1} Y+\hat{A}_{2} X Y+\hat{A}_{4} X^{2} Y+\tilde{A}_{6} Y^{3}, \\
& \dot{y}=\left(z_{5}-z_{4}\right) X^{2}+X^{3}-\hat{B}_{0} Y^{2}+\tilde{B}_{5} X Y^{2},
\end{aligned}
$$

where $\hat{A}_{1}=\tilde{A}_{1}+\tilde{A}_{2} z_{5}+\tilde{A}_{4} z_{5}^{2}, \hat{A}_{2}=\tilde{A}_{2}+2 \tilde{A}_{4} z_{5}$ and $\hat{B}_{0}=B_{0}-B_{5} z_{5}$. Note that in view of Lemma 9 we have that $\hat{A}_{1} \neq 0$. Now we introduce a scaling of time of the form $\tau=t / \hat{A}_{1}$ and we have that system (21) becomes

$$
\begin{aligned}
& \dot{x}=Y+\bar{A}_{2} X Y+\bar{A}_{4} X^{2} Y+\bar{A}_{6} Y^{3}, \\
& \dot{y}=\frac{z_{5}-z_{4}}{\hat{A}_{1}} X^{2}+\frac{1}{\hat{A}_{1}} X^{3}-\bar{B}_{0} Y^{2}+\bar{B}_{5} X Y^{2},
\end{aligned}
$$

where

$$
\bar{A}_{2}=\frac{\hat{A}_{2}}{\hat{A}_{1}}, \bar{A}_{4}=\frac{\hat{A}_{4}}{\hat{A}_{1}}, \bar{A}_{6}=\frac{\tilde{A}_{6}}{\hat{A}_{1}}, \bar{B}_{5}=\frac{\hat{B}_{5}}{\hat{A}_{1}}, \bar{B}_{8}=\frac{\tilde{B}_{8}}{\hat{A}_{1}} .
$$

Note that $(0,0)$ is a nilpotent singular point and that system $(22)$ is precisely system (14) with

$$
\begin{array}{r}
A(X, Y)=\bar{A}_{2} X Y+\bar{A}_{4} X^{2} Y+\bar{A}_{6} Y^{3}, \\
B(X, Y)=\frac{z_{5}-z_{4}}{\hat{A}_{1}} X^{2}+\frac{1}{\hat{A}_{1}} X^{3}-\bar{B}_{0} Y^{2}+\bar{B}_{5} X Y^{2} .
\end{array}
$$

The solution $Y+f(X)$ of $Y+A(X, Y)=0$ is $f(X)=0$. Then

$$
F(X)=B(X, 0)=\frac{z_{5}-z_{4}}{\hat{A}_{1}} X^{2}+\frac{1}{\hat{A}_{1}} X^{3}
$$

and $G(X)=\left(A_{2}+2 A_{4} X-2 B_{0} Y+2 B_{5} X Y\right)_{Y=0}=0$. So we are under the assumptions of Lemma 11 with $m=2$ and $a=\left(z_{5}-z_{4}\right) / \hat{A}_{1}$. In view of Lemma 11 we have that it is a cusp. This proves statement (d).

If $B_{4} \neq 0$ and $D_{0}<0$ then $G(x)=0$ has three simple real solutions: $z_{6}, z_{7}$ and $z_{8}$, and so by Lemma 10 they can only be saddles or centers. This proves statement (e).

If $B_{4} \neq 0$ and $D_{0}>0$ then $G(x)=0$ has a unique simple real solution that we call $z_{9}$. Again, by Lemma 10 it can only be a saddle or a center. This proves statement (f).

Note that in view of Lemma 12 all singular points of system (3) with $a_{3}=0$ have either topological index -1 (and then they are saddles), or topological index 0 (and they the can only be cusps), or topological index 1 (and they can only be centers). Now we use this information 
to obtain a restriction on the shape of the singular points when $f_{1}=0$ and $f_{2}=0$ are both limit cycles of system (3) with $a_{3}=0$.

To prove Corollary 14 we shall need the following result. For a proof of it see, for instance, Proposition 6.26, Example 6.17 and Proposition 6.7 in $[2]$.

Lemma 13. In the interior of a limit cycle there must be singular points so that the sum of their topological indices is one.

Corollary 14. System (3) with $a_{3}=0$ and having $f_{1}=0$ and $f_{2}=0$ as limit cycles can have only three real singular points: $(-x, 0),(0,0)$ and $(x, 0)$. Moreover, $( \pm x, 0)$ are centers and $(0,0)$ is a saddle.

Proof. In order to prove that $f_{1}=0$ and $f_{2}=0$ be limit cycles we must have that $a_{0} \neq 0, \Delta_{0}<0$ and $\Delta_{1}<0$, see Proposition 8. Also inside the proof of Proposition 8 we show that the singular points of system (3) are of the form $(z, 0)$ with $F(z)=0$. In view of Lemma 5 , system (3) with $a_{3}=0$ is symmetric with respect to the $y$-axis. So, if system (3) has a unique singular point then this singular point must be the origin $(0,0)$. By Lemma 13 it must have topological index one and by the statements of Lemma 12 it must be a center, and consequently by Lemma 6 the circle surrounding this singular point cannot be a limit cycle. So, statements (a), (c) and (f) of Lemma 12 cannot hold.

Now assume that there are only two singular points of system (3) with $a_{3}=0$. By the symmetry they are of the form $( \pm x, 0)$ and both of them must have the same index. In view of Lemma 12 the only possibility is statement (b) (because in statement (d) both singular points have different index). But statement (b) is also not possible, because both points have topological index either 1 , or -1 , so in view of Lemma 13 in at least inside the region delimited by one of the circles $f_{1}=0$ or $f_{2}=0$ there is a unique singular point, with topological index 1. Therefore, by the statement (b) of Lemma 12 such a singular point must be a center, and consequently by Lemma 6 that circle cannot be a limit cycle. So statement (b) cannot hold.

In short, the unique possibility is that statement (e) of Lemma 12 holds, i.e. there are three singular points of system (3) with $a_{3}=0$. By symmetry these singular points must be of the form $( \pm x, 0)$ and $(0,0)$. Moreover, $( \pm x, 0)$ have the same index. In view of statement (e) of Lemma 12 and the existence of the two invariant circles which are periodic orbits, we must have that $( \pm x, 0)$ are centers and $(0,0)$ is a saddle. This completes the proof of the corollary.

Now we study the singular points of system $(3)$ when $a_{3} \neq 0$. In this case, in view of Lemma 9, the singular points can be either hyperbolic, 
or semi-hyperbolic. More concretely, let $\tilde{x}$ be a double zero of $F(x)$, then $B_{1}+2 B_{3} \tilde{x}+3 B_{4} \tilde{x}^{2}=0$, and the singular point $(\tilde{x}, 0)$ is either a saddle, or a node, or a saddle-node. Now we introduce the notation

$$
\begin{aligned}
& H_{0}(x)=\left(A_{1}+A_{2} x+A_{4} x^{2}\right)\left(B_{1}+2 B_{3} x+3 B_{4} x^{2}\right), \\
& H_{1}(x)=\frac{\left(B_{2}-A_{3} x+B_{2} x^{2}\right)^{2}}{4}+H_{0}(x) .
\end{aligned}
$$

If $\tilde{x}$ is a simple zero of $F(x)$, then the singular point $(\tilde{x}, 0)$ is a saddle if $H_{0}(\tilde{x})>0$, a node if $H_{1}(\tilde{x}) \geq 0$ and a focus if $H_{1}(\tilde{x})<0$.

Lemma 15. System (3) with $a_{3} \neq 0, a_{0} \neq 0 \Delta_{0}<0, \Delta_{1}<0$ has the following singular points.

(a) If $B_{4}=0$ and $B_{3}=0$, then if $B_{1}=0$ it has no singular points, and if $B_{1} \neq 0$ it has the unique singular point $\left(z_{0}, 0\right)$ where $z_{0}$ is given in (15). It is a saddle if $H_{0}\left(z_{0}\right)>0$, a node if $H_{1}\left(z_{0}\right) \geq 0$ and a focus if $H_{1}\left(z_{0}\right)<0$.

(b) If $B_{4}=0$ and $B_{3} \neq 0$, then it has the two singular points $\left(z_{1}, 0\right)$ and $\left(z_{2}, 0\right)$ with $z_{1}$ and $z_{2}$ given in (16) when either (II) holds, or (III) holds with $B_{1}^{2}-4 B_{0} B_{3}>0$. For $j=1,2, z_{j}$ is a saddle if $H_{0}\left(z_{j}\right)>0$, a node if $H_{1}\left(z_{j}\right) \geq 0$ and a focus if $H_{1}\left(z_{j}\right)<0$. If (III) holds with $B_{1}^{2}-4 B_{0} B_{3} \leq 0$, then either $f_{1}=0$ or $f_{2}=0$ cannot be a limit cycle.

(c) If $B_{4} \neq 0$ and $D_{0}=D_{1}=0$, then $F(x)=0$ has a unique triple solution $z_{3}$. The singular point $\left(z_{3}, 0\right)$ is semi-hyperbolic, and consequently it is either a saddle, a node, or a saddle-node.

(d) If $B_{4} \neq 0, D_{0}=0$ and $D_{1} \neq 0$, then $F(x)=0$ has two distinct real solutions (one simple $z_{4}$ and one double $z_{5}$ ). The singular point $\left(z_{4}, 0\right)$ is a saddle if $H_{0}\left(z_{4}\right)>0$, a node if $H_{1}\left(z_{4}\right) \geq 0$ and a focus if $H_{1}\left(z_{4}\right)<0$. The singular point $\left(z_{5}, 0\right)$ is semihyperbolic.

(e) If $B_{4} \neq 0$ and $D_{0}>0$, then $F(x)$ has three distinct simple real solutions $z_{6}, z_{7}, z_{8}$. The singular point $\left(z_{j}, 0\right)$ for $j=6,7,8$ is either a saddle if $H_{0}\left(z_{j}\right)>0$, a node if $H_{1}\left(z_{j}\right) \geq 0$, and a focus if $H_{1}\left(z_{j}\right)<0$.

(f) If $B_{4} \neq 0$ and $D_{0}<0$, then it has a unique simple real solution $z_{9}$. The singular point $\left(z_{9}, 0\right)$ is a saddle if $H_{0}\left(z_{9}\right)>0$, a node if $H_{1}\left(z_{9}\right) \geq 0$, and a focus if $H_{1}\left(z_{9}\right)<0$.

Proof. It follows by direct calculations. Note that when $B_{4}=0$ equation $F(x)=0$ becomes the quadratic equation $B_{3} x^{2}+B_{1} x+B_{0}=0$. If $B_{3}=0$ then if $B_{1}=0$ it has no solutions and if $B_{1} \neq 0$ then the solution is the $z_{0}$ given in (15). It is a simple solution of $F(x)=0$ 
and so it is hyperbolic. Therefore, by the explanation before the statement of Lemma 15 we have that it is a saddle if $H_{0}\left(z_{0}\right)>0$, a node if $H_{1}\left(z_{0}\right) \geq 0$ and a focus if $H_{1}\left(z_{0}\right)<0$. This proves statement (a).

If $B_{4}=0$ and $B_{3} \neq 0$ then $F(x)=0$ has the two solutions given in (16). Proceeding as in statement (b) in the proof of Lemma 12 we conclude that only when condition (III) holds with $B_{1}^{2}-4 B_{0} B_{3} \leq 0$ both solutions collide or disappear, so never they are simple solutions, but in this case the same arguments imply that either $f_{1}=0$, or $f_{2}=0$ cannot be a limit cycle. On the other cases, $z_{1}$ and $z_{2}$ are hyperbolic. Therefore, for $j=1,2, z_{j}$ is a saddle if $H_{0}\left(z_{j}\right)>0$, a node if $H_{1}\left(z_{j}\right) \geq 0$, and a focus if $H_{1}\left(z_{j}\right)<0$. This proves statement (b).

If $B_{4} \neq 0$ and $D_{0}=D_{1}=0$, we have a unique triple solution $z_{3}$ for $F(x)=0$. In this case the singular point $\left(z_{3}, 0\right)$ is semi-hyperbolic and by Theorem 2.19 in [2] it is either a saddle (if it has index -1), a node (if it has index 1), or a saddle-node (if it has index 0 ). This proves statement (c).

If $B_{4} \neq 0, D_{0}=0$ and $D_{1} \neq 0$, we have a simple solution $z_{4}$ and a double solution $z_{5}$ for $F(x)=0$. The singular point $\left(z_{5}, 0\right)$ is semihyperbolic and thus it is either a saddle, a node, or a saddle-node. The singular point $\left(z_{4}, 0\right)$ is simple and it can be a saddle if $H_{0}\left(z_{4}\right)>0$, a node if $H_{1}\left(z_{4}\right) \geq 0$, and a focus if $H_{1}\left(z_{4}\right)<0$. This proves statement (d).

The proof of statements (e) and (f) are similar to the previous ones.

\section{Proof of Theorems 3 And 4}

In this section we prove Theorems 3 and 4 separately.

Proof of Theorem 3. In view of Corollary 14 the unique possible distribution for the singular points is $( \pm x, 0)$ and $(0,0)$, being $( \pm x, 0)$ centers and $(0,0)$ a saddle.

Assume first we are under the assumptions (III). By Lemma 13 each circle $f_{1}=0$ and $f_{2}=0$ surrounds a center, and by Lemma 6 these circles cannot be limit cycles.

Now assume we are on the assumptions (II). All the three singular points must be contained in the limited region of the circle $f_{2}=0$, otherwise that circle would be inside the period annulus of a center, and consequently it would not be a limit cycle (see Lemma 6). Since $f_{1}=0$ must be a limit cycle, the orbit around it must spiral, but this is not possible due to the symmetry with respect to the $y$-axis (see Lemma 5). 
Proof of Theorem 4. Assume that $f_{1}=0$ and $f_{2}=0$ are limit cycles. Then by Theorem 3 and the arguments in the introduction $a_{0} a_{3} \neq 0$. Since they are periodic orbits in view of Proposition 8 we must have $\Delta_{0}<0$ and $\Delta_{1}<0$.

If (II) holds and $B_{4}^{2}+B_{3}^{2}+B_{1}^{2}=0$ then, by Lemma 15 (a) there are no singular points and thus $f_{1}=0$ and $f_{2}=0$ cannot be limit cycles. So, if we are under the assumptions (II) we must have $B_{4}^{2}+B_{3}^{2}+B_{1}^{2} \neq 0$. On the other hand, if (III) holds with $B_{4}=0$, and either $B_{3}=0$ or $B_{3} \neq 0$ with $B_{1}^{2}-4 B_{0} B_{3} \leq 0$, then by Lemma $15(\mathrm{~b}), f_{1}=0$ and $f_{2}=0$ cannot be limit cycles. So in order that both $f_{1}=0$ and $f_{2}=0$ are limit cycles if (III) holds and $B_{4}=0$ we must have $B_{3} \neq 0$ and $B_{1}^{2}-4 B_{0} B_{3}>0$. This proves the "only if" part of Theorem 4 .

Now assume that $a_{0} a_{3} \neq 0, \Delta_{0}<0, \Delta_{1}<0$ and either $B_{4}^{2}+B_{3}^{2}+B_{1}^{2} \neq$ 0 if (II) holds, or (III) holds and $B_{4}=0, B_{3} \neq 0$ and $B_{1}^{2}-4 B_{0} B_{3}>0$. In view of Proposition 8 we have that $f_{1}=0$ and $f_{2}=0$ are periodic orbits. Moreover, since $a_{3} \neq 0$ by Lemma 15 all singular points are either hyperbolic or semi-hyperbolic, and thus they must be either a focus, or a node, or a saddle, or a saddle-node (see Lemma 2.19 in $[2])$. In order to prove that $f_{1}=0$ and $f_{2}=0$ are limit cycles it only remains to prove that they are isolated in the set of all periodic orbits of the differential system. We proceed by contradiction. Assume that they are non-isolated. Since the Poincaré map defined in a transversal section in each of the two circles is analytic (the differential system is polynomial and consequently analytical), it must be the identity. In short, we have a continuum of periodic orbits surrounding a focus, a node, a saddle or a saddle-node, but this is a contradiction because it cannot exist the closest periodic orbit of this continuum either to the focus, or to the node, or to the saddle, or to the saddle-node, because if such closest periodic orbit $\gamma$ exist applying again the argument of the analyticity of the Poincaré map defined in a transversal section to $\gamma$ it follows that $\gamma$ is not the closest periodic orbit to the focus, to the node, to the saddle or to the saddle-node, a contradiction. This completes the "if" part of Theorem 4.

\section{ACKNOWLEDGEMEnTs}

The first author is partially supported by a MINECO grant number MTM2014-53703-P, and an AGAUR (Generalitat de Catalunya) grant number 2014SGR 1204 . The second author is partially supported by a MINECO grant MTM2013-40998-P, an AGAUR grant 2014SGR 568, and two grants FP7-PEOPLE-2012-IRSES numbers 316338 and 
318999. The third author is partially supported by FCT/Portugal through the project UID/MAT/04459/2013.

\section{REFERENCES}

[1] A.F. AndReEv, Investigation of the behaviour of the integral curves of a system of two differential equations in the neighbourhood of singular points, Trans. Amer. Math. Soc. 8 (1958), 183-207.

[2] F. Dumortier, J. Llibre and J.C. Artés, Qualitative theory of planar differential systems, Universitext, Springer-Verlag, 2006.

[3] J. Llibre And G. Swirszcz, Classification of quadratic systems admitting the existence of an algebraic limit cycle, Bull. Sci. Math. 131 (2007) 405-421.

[4] J. Llibre, R. Ramírez, V. Ramírez and N. Sadovskaia, The 16-th Hilbert problem restricted to circular algebraic limit cycles, preprint, 2015.

[5] V.A. LunKEVICH AND K. S. SiBIRSKII, Integrals of a general quadratic differential system in cases of a center, Differential Equations 18 (1982), 563-568.

${ }^{1}$ Departament de Matemàtica, Universitat de Lleida, Avda. Jaume II, 69; 25001 Lleida, Catalonia, Spain

E-mail address: gine@matematica.udl.cat

2 Departament de Matemàtiques, Universitat Autònoma de Barcelona, 08193 Bellaterra, Barcelona, Catalonia, Spain

E-mail address: jllibre@mat.uab.cat

3 Departamento de Matemática, Instituto Superior Técnico, Av. Rovisco Pais 1049-001, Lisboa, Portugal

E-mail address: cvalls@math.ist.utl.pt 